\title{
ESTUDIO DE 50 BRONCONEUMONIAS POST SARAMPIONOSAS EN LACTANTES
}

\author{
Drs. ABRAHAM OYANEDEL y JORGE E. HOWARD \\ Cátedra de Pediatría del Prof. Dr. Aníbal Ariztia. \\ Hospital Luis Calvo Mackenna.
}

\section{INTRODUCCIÓN}

Por haber ingresado durante el año 1958 al Servicio de Recepción de 1a Primera Infancia del Hospital Luis Calvo Mackenna numerosos lactantes con bronconeumonia, decidimos dividir nuestro material en 2 grupos: $1^{\circ}$ los que en un plazo no mayor a 12 dias antes del ingreso habian iniciado su rash sarampionoso y 2 aquellas lactantes que presentando bronconeumonía no habían tenido sarampión. El motivo que nos impulsaba era estudiar detalladamente estos 2 grupos para comparar y tratar de obtener una visión clara de las posibles diferencias que justificaran el seguir manteniendo el diagnóstico de bronconeumonía post sarampionosa como una realidad diferente de la bronconeumonía bacteriana del lactante hospitalizado, vale decir, del lactante distrófico.

\section{MATERIAL $\mathrm{x}$ MÉTODO}

Tomamos para este estudio 50 lactantes que sin previa selección ingresaron al Servicio de Recepción Primera Infancia entre los meses de Marzo a Diciembre de 1958, y que en un lapso no mayor de 12 días antes del ingreso habían presentado la iniciación de su rash sarampionoso y que examinados por 2 médicos, ambos establecieron clínicamente el diagnóstico de bronconeumonía, diagnóstico que fuera confirmado a rayos por el radiólogo, en algunos casos con radiografía y en todos con radioscopía. A estos lactantes se les sometió tratamiento, cuidados de enfermería y dietética semejante. En todos se llevó una hoja especial en que diariamente se anotó la variación de la fiebre, estado general y examen pulmonar anotando los cambios de: sonoridad, estertores, crépitos y subcrépitos.

Fuera de la radioscopía se practicaron hemogramas $y$ examen de orina que se repitieron a los 7 días.
Como control se tomaron 50 lactantes hospitalizados en el mismo servicio con el diagnóstico de bronconeumonía que no había presentado sarampión, y en los cuales se llevó Ia misma metódica ya descrita.

\section{RESULTADOS}

1. Estado nutritivo: Vemos en el cuadro N? 1 que nuestro material en ambos grupos es semejante, siendo la gran mayoría distróficos. Para mayor comparabilidad dividimos a los distróficos en 3 grados de acuerdo con el porcentaje del peso ideal que debieron tener, y en las distintas subdivisiones, se obtuvieron resultados aceptables.

CUADRO NQ 1

BSTADO NUTRITTVO DE SO LACTANTES CON BRONCONFUMONIA POST SARAMIIONOSA Y 50 CONTROLES

\begin{tabular}{lcccr}
\hline & Eutrofia & 1 & $\begin{array}{c}\text { Distrolis } \\
2\end{array}$ & 3 \\
\hline Estudio & 11 & 23 & 12 & 4 \\
\hline Control & 10 & 23 & 12 & 6 \\
\hline TOTAL & 21 & 46 & 28 & 10 \\
\hline
\end{tabular}

2. Edad: En el cuadro No 2 podemos ver que también ambos grupos son semsiblemente iguales y que la bronconeumonía post sarampionosa la observamos más frecuentemente en los lactantes mayores del año.

CUADRO N9 2

EDAD EN MEARS DE 30 I-ACTANTBS CON BRONCONEUMONIA POST SARAMPIONOSA Y 50 CONTROLES

\begin{tabular}{lccc}
\hline & $0-5 \mathrm{~ms}$. & $6-11 \mathrm{~ms}$ & $12-24 \mathrm{~ms}$ \\
\hline Estudis & 3 & 14 & 33 \\
\hline Control & 4 & 18 & 30 \\
\hline TOTAL & 7 & 30 & 63 \\
\hline
\end{tabular}

3. Comienzo: En el cuadro N9 3 vemos que la mayoría de las bronconeumonías 
ingresan al hospital después de los 3 días de iniciado el rash.

CUADRO No 3

DIAG DESPUEg DEL gARAMPION BN QUE gE INICIO LA BRONCONGUMONIA DE 50 LACTANTWS EAN ESTUDIO

\begin{tabular}{lccccc}
\hline & -3 dias & $4-7$ dies & $8-12$ dias & Total \\
\hline No de cesos & 9 & 26 & 15 & 50 \\
\hline
\end{tabular}

4. Sintomatología clínica: En el cuadro $N^{\circ} 4$ vemos que hubo un mayor número de lactantes en estudio que presentaron fiebre más prolongada y que un número pequeño en ambos grupos evolucionó en forma afebril.

Ell cuadro también nos muestra que la sintomatología física fué algo más prolongada en el grupo en estudio.

CUADRO No 4

DURACION DE IA FIRBRE $Y$ STVTOMAS PULMONARES DE 50 LACTANTES CON BRONCONEUMONIAS POS'T SARAMPIONOSAS $Y$ 50 CONIROLES *

\begin{tabular}{|c|c|c|c|c|c|c|}
\hline & \multicolumn{2}{|c|}{ 1-3 diag } & \multicolumn{2}{|c|}{ 4-7 d1as } & \multicolumn{2}{|c|}{ más 7 días } \\
\hline & $\begin{array}{l}\text { Estu- } \\
\text { d1o }\end{array}$ & $\begin{array}{l}\text { Can- } \\
\text { trol }\end{array}$ & $\begin{array}{l}\text { Estu- } \\
\text { d10 }\end{array}$ & $\begin{array}{l}\text { Con- } \\
\text { trol }\end{array}$ & $\begin{array}{l}\text { Estu= } \\
\text { dIo }\end{array}$ & $\begin{array}{l}\text { Con- } \\
\text { troI }\end{array}$ \\
\hline Fiebre & 18 & 26 & 10 & 9 & 17 & 9 \\
\hline $\begin{array}{l}\text { Bintomas } \\
\text { pulmonsres }\end{array}$ & - & 2 & $2 B$ & 32 & 24 & 16 \\
\hline
\end{tabular}

- Afebrll evol. 5 del g. estudio y 6 del g. contral.

5. Sintomatoloqía radiológica: En el cuadro No 5 estudiamos Ia sintomatología radiológica en los primeros 2 días de ingreso al hospital y vemos que en el grupo en estudio 14 presentaban lesiones unilaterales $y$ en $\mathbf{3 1}$ bilaterales, $\mathrm{y}$ de estas $\mathbf{4 5}$. 19 eran difusas. En el grupo control 12 eran unilaterales y 33 bilaterales, pero de estas 45,31 eran difusas.

\section{CUADRO N9 5}

SINTOMATOLOGTA RADIOLOCTCA AL INGRESO DE 45 LACTANTES CON BRONCONEUMONTA POST BARAMPIONOSA $Y$ 45 CONIROLES

\begin{tabular}{lccccc}
\hline & \multicolumn{2}{c}{ Unllateral } & \multicolumn{2}{c}{ Bllatetal } \\
\hline & D!fues & Ctreun. & & Ditusa & Clreun. \\
\hline Estudio & 3 & 11 & & 16 & 15 \\
\hline Control & 4 & 8 & 27 & 6 \\
\hline TOTaL & 7 & 19 & 4 & 21 \\
\hline
\end{tabular}

6. Complicaciones: En el cuadro No 6 vemos que hay mayor número de complicaciones en el grupo en estudio $y$ éstas son especialmente de las vias aéreas superiores. También en este grupo 2 bronconeumonías evolucionan hacia la formación de abscesos pulmonares.

\section{CUADRO No 6}

COMPLICACIONES EN HL CURSO DE LA HOSPTTALIZACION DE 50 BRONCONEUMONIAS POST-SARAMPIONOSAR $T$ 50 CONTROLES *

\begin{tabular}{lcc}
\hline Complieaclones & Estudio & Control \\
\hline Faringitts & 9 & 4 \\
\hline Bronquitis & 5 & 2 \\
\hline Iaringitis & 4 & 0 \\
\hline Otitls & 7 & 0 \\
\hline Plurlas & 7 & 6 \\
\hline N. Bronconeninonfa & 4 & 2 \\
\hline Absceso Pulm. & 2 & 0 \\
\hline TOTAL & 38 & 14 \\
\hline Falleclios & 2 & 0 \\
\hline
\end{tabular}

7. Tratamiento: Se usaron en general 2 Planes de tratamiento en ambos grupos.

PIan A. Penicilina o Penicilina y Estreptomicina a la dosis de 200.000 unidades y $50 \mathrm{mg}$. respectivamente por kilogramo de peso por día.

Plan B. Antibiótico de amplio espectro (Oxitetraciclina o Tetraciclina) a la dosis de $50 \mathrm{mg}$. por $\mathrm{kg}$. de peso y por día.

\section{CUADRO NP 7}

CUADRO COMPARATIVO DE LOS RESULTADOS DEL TRATAMTRNTO FN 50 LACTANTES CON RRONCONEUMONIA POST SARAMLPION Y 50 CONTROLFS

\begin{tabular}{|c|c|c|c|c|c|c|}
\hline & \multicolumn{3}{|c|}{ Plan A } & \multicolumn{3}{|c|}{ Plan $\mathrm{B}$} \\
\hline & No & Exitos & $\begin{array}{l}\text { Fraceso } \\
\text { (camb. } \\
\text { droga) }\end{array}$ & NP & Exitos & $\begin{array}{l}\text { Fracaso } \\
\text { (camb. } \\
\text { droga) }\end{array}$ \\
\hline Estudio & 33 & $20^{\circ}$ & 13 & 16 & I3 & 3 \\
\hline Control & 35 & 25 & 10 & 15 & 10 & 5 \\
\hline
\end{tabular}

Con el Plan A fueron tratados 34 Jactantes del grupo en estudio y 35 controles, observándose en el cuadro No 7 que de los 34 casos en estudio, 20 curaron completamente, $\mathrm{y}$ en 13 después de 7 días de tratamiento hubo que cambiar de antibiótico a uno de más amplio espectro. 1 falleció a los 2 días de hospitalización. En los controles se trataron 35 lactantes, 25 meioraron $y$ en 10 hubo que cambiar a otro antibiótico de amplio espectro.

Con el Plan B se trataron 16 lactantes del grupo en estudio de los cuales 13 me- 
joraron, $y$ en 3 hubo de cambiarse el antibiótico después de 7 días. De los controles se trataron 15 con meioría en $10 \mathrm{y}$ fracaso del tratamiento en 5.

8. Duración de la hosnitalización: Podemos ver en el cuadro NQ 8 aue los casos en estudio en general estuvieron hospitalizados durante un período más largo que Ios controles.

CUADRO NQ 8

ALTA FN DIAS DE 50 LAGTANTFE CON RRCONCONEUMONIA BARAMPIONOSA $T$ 50 CONTROLES *

\begin{tabular}{|c|c|c|c|}
\hline & $-10 \mathrm{dtes}$ & $11-20$ d1es & 21 đIiAs , mas \\
\hline Fstueio & B & 16 & 24 \\
\hline Control & 17 & 16 & 15 \\
\hline TOTAL & 28 & 34 & 39 \\
\hline
\end{tabular}

9. Hemograma: En el cuadro No 9 vemos nı̣ la fórmula fúé sensiblemente iguales en ambos grupos.

CTADRO NQ 9

CIPRA DE LFUCOCTTOS EN LACTANTES TON MRAV. CONEUMONIA POS'T AARAMPTONOSA $Y$ LACTANTES GRUPO TBSTIGO

\begin{tabular}{lccc}
\hline & $8-13 \mathrm{mll}$ & $13,100-18 \mathrm{mll}$ & msa $18 \mathrm{mil}$ \\
\hline Estudlo & 30 & 12 & 5 \\
\hline Testlgo & 31 & 10 & 8 \\
\hline
\end{tabular}

\section{COMENTARIO}

Está claro que nuestros 2 grupos, es decir el gruoo en estudio, aquellos lactantes aue 12 días antes presentaron saramnión $\checkmark$ el grupo control aauellas bronconeumonias aue evolucionaron en lactantes aue nn hahían tenido saramoión son comnarabJes. Los cuadros N.os 1 y 2 nos indican aue tanto desde el punto de vista del estado nutritivo como de la edad. ambos erupos son semejantes. Además los criterios establecidos nara confirmar el diagnóstico de bronconeumonia fueron nrecisos. $y$ si en la exposición fíguran sólo 45 lactantes ron estudio radiolónico. se debe a que en los 5 restantes en cada grupo. exte ex amen no se practicó al ingreso sino días mis tarde, pero de todas maneras en todos fué positivo. Insistimos en este hecho. de un examen de ravos positivo. nor cuanto esnecialmente en los nrimeros días después del rash puode una bronauiolitis confundirse con una bronconeumonía $y$ de esta manera aumentar la incidencia de esta complicación y disminuir su gravedad. Igual que lo confirmado por la mayoría de los autores que han estudiado el tema, hemos visto que esta complicación se presenta con mayor frecuencia en los 7 días siguientes a la iniciación de rash, como sucedió en las $2 / 3$ partes de nuestros casas.

En nuestro Hospital ${ }^{1}$, Martínez, Trautman y Wiedenslaufer ${ }^{1}$, estudiando los resultados de un tratamiento standard en la bronconeumonía post sarampionosa, también anotan que poco más de las $2 / 3$ partes de su material presentan esta complicación en la primera semana de la iniciación del rash, pero a diferencia de lo observado por nosotros, encontraron una mayor proporción de casos iniciados precozmente, es decir en los primeros 3 días, lo cual nos está mostrando que el sarampión presenta diverso genio epidémico y que si de año a año las complicaciones son de frecuencia diferentes, también en distintas epidemias las mismas complicaciones pueden presentarse en momentos diversos.

No pudimos comfirmar lo aseverado por diversos autores, que los casos de mayor gravedad corresponden a los que precozmente iniciaron la complicación pulmonar en relación al exantema, y que para algunos deberian su mayor gravedad al hecho de ser una complicación virósica, por el virus del sarampión, de forma intersticial y no polimicrobiana como se acepta hoy, que es la etiología de la mavoría de las bronconeumonías post saramoionosas.

Los tratados clásicos describer la sintomatología de la bronconeumonía post sarampionosa igual a la de la bronconeumonía por focos diseminados y que en unos momentos se presenta la forma lobar o pseudolobar. En esta revisión también encontramos ésto, es decir, la trecuencia del desplazamiento de los focos de un día a otro can la consiguiente variabilidad de los soplos que fueron más o menos intensos y rodeados generalmente de estertores subcrepitantes finos.

Lo que nos llamó la atención y aue está expresado claramente en el cuadro $N$ Q 4 fué que tanto la fjebre como los signos físicas pulmonares propios de la bronconeumonía, persisten más tiempo en los lactantes del grupo en estudio que en los controles o sea que en el lactante distró- 
fico, la bronconeumonía post sarampionosa, evoluciona en un plazo mayor que la bronconeumonía bacteriana del lactante. Esto está en contraposición con 1o aseverado por Stokes ${ }^{2}$ en el tratado de Michell y Nelson, quien encuentra que la evolución de la bronconeumonía post sarampionosa es igual a las bronconeumonías bacterianas, y esta diferencia seguramente se debe al material distinto, por cuanto ya hemos visto que el nuestro, está formado en su gran mayoría por lactantes distróficos.

En la radiología nos hemos encontrado con un hecho que no nos podemos explicar claramente $\mathrm{y}$ es que contrariamente a lo manifestado por los diversos autores, en nuestro material de bronconeumonfas post sarampionosas han primado las lesiones circunscritas, Io cual no sucede en el gruno control. Martínez y colaboradores en el trabaio ya citado encuentran que cuando la lesión era unilateral existía una mayoría de lesiones circunscrita, pero que en el $\mathbf{9 0 \%}$ de los casos en aue a ravos se comprobaba la bilateralidad de las lesiones. estas eran difusas.

El hemograma de Ia bronconeumonía post sarampionosa del lactante. no es característico. no observando la leucobenia del sarampión, sino la leucocitosis propia de la infección microbiana y no encontramos relación entre él y la evolución de los enfermos.

En lo referente al tratamiento observamos que no se nota una diferencia apreciable entre el grupo en estudio y el gruno control, en cuanto al tiembo en que se demnra en establecerse la meioría clínica. De nuestro material deducimos que el tratamiento aue da mavores espectativas de mejoría clínica y radiológica en un vlazo más corto es el empleo de antibióticos de amolio espectro, por cuanto de 16 enfermos tratados con oxitetraciclina o tetraciclina en sólo 3 hubo fracaso de la droga al cabo de 1 semana, en tanto aue de 33 tratados con Penicilina o Penicilina más Estreptomicina en $13(40 \%)$ al cabo de 7 días hubo que cambiarse precisamente a un antibiótico de amplio espectro.

Es de señalar, tal como ya to han publicado otros autores nacionales (Martínez y colaboradores y Undurraga y Banfi $^{8}$ que la mortalidad de Ia bronconeumonía post sarampionosa es baja, a pesar de tratarse de un material de niños distrófi- cos. Recalcamos este hecho por cuanto algunos autores extranjeros (Bazan, 1955) ${ }^{4}$ muestran porcentajes elevadas que llegan desde el $100 \%$ en los menores de 6 meses a un $38 \%$ en el grupo de 1 a 2 años. De los casos nuestros que fallecieron uno de ellos falleció a $\operatorname{los} 2$ días de tratamiento de una bronconeumonía aparecida al segundo día del comienzo del rash. Se trataba de un lactante de 1 año 2 meses, distrófico. que ingresó de suma gravedad. EI otro fallecido fué un lactante de $\mathbf{1 1}$ meses de edad distrófico que había iniciado su bronconeumonía 7 días después del rash sarampionoso y que después de haber sanado de su bronconeumonia con el Plan A, a los 19 días del ingreso presenta una nueva bronconeumonía de la cual fallece 7 días después. A la Anatomía Patológica se comprobó una bronconeumonía abcediente con estafilococo.

\section{RESUMEN Y CONCLUSIONES}

El lactante distrófico hospitalizado por una bronconeumonía aparecida en los 12 días siguientes a la iniciación del rash propio del sarampión comparado con un lactante de la misma edad y mismo erado de distrofia con una bronconeumonía bacteriana no precedida de saramoión presenta ciertas características comunes y diferenciales.

1. Aparece la bronconeumonía post sarampionasa en la mayoría de los casos en los 7 días que siguen a la iniciación del rash y no se encuentra mayor gravedad a mavor prioridad de comienzo de la complicación pulmonar.

2. La sintomatología clínica es semejante en ambos, pero tanto la duración de la fiebre como de los signos pulmonares es más prolongada en los que han hecho sarampión.

3. A la radiología predomina las lesiones circunscritas en los lactantes del grupo estudio, siendo al contrario más đifusas en los controles.

4. El hemograma al ingreso y en su evolución es semejante en ambos.

5. Las bronconeumonías post sarampionosas presentan más complicaciones, especialmente de las vías aéreas altas y también más complicaciones pulmonares en nuestro material, 2 abscesos pulmonares. 
6. La mortalidad es baja. $4 \%$ en nuestra serie.

7. EI tratamiento de Ia bronconeumonía post sarampionosa a esta edad debe ser hecho a base de antibióticos de amplio espectro que garantizan mejores expectativas de éxito.

8. Por todos estos considerando creemos que debe mantenerse el diagnóstico de bronconeumonía post sarampionosa, no como entidad diferente de la bronconeumonía bacteriana del lactante, sino como un indice de mayor gravedad en el pronóstico.

\section{SUMMARY AND CONCLUSIONS}

The authors compares dystrophic infants hospitalized with bronchopneumonia which appeared in the first 12 days after the rash of measles, with infants of the same age and the same degree of dystrophy but with a bacterial bronchopneumonia.

1) Bronchopneumonia subsequent to measles appears in the majority of the cases during the seventh day after the beginning of the rash. When puimonary complications apears earlier, no greater severity is found.

2) Clinical symptoms are similar in both groups, but fever and pulmonary signs lasts for a longer period in those who have had measles.

3) On X-ray examination, circumscribed lesions prevailed among the measles group, and more diffuse ones, among the control groups.

4) On admission and during the evolution, hemogram is similar in both groups.

5) Bronchopneumonias subsequent to measles show complications, especially of the upper respiratory tract.

6) The mortality rate is low, $4 \%$ in our series.

7) The treatment of bronchopneumonia subsequent to measles at this age. has to be based on a broad-spectrum antibiotics which offer better prospects of success.

8) We believe for all these considerations that the diagnosis of bronchopneumonia subsequent to measles ought to be maintained, not as a different entity from the bacterial bronchopneumonia but as an index of a more serious prognosis.

\section{ZUSAMMENFASSUNG UND SCHLUSSFOLGERUNGEN}

Wenn man eine $n$ dystrophischen Saeugling, der wegen einer in den ersten 12 Tagen nach dem Beginn des eigentli$\mathrm{c} h$ e $n$ Masemexanthems aufgetretenen Bronchopneumonie ins Krankenhaus aufgenommen wurde, mit einem Saeugling in gleichen Alter, mit gleich starker Dystrophie und mit einer bakteriellen Bronchopneumonie ohne vorhergegangene Masern vergleicht, so zeigen sich gewisse, gemeinsame und unterschiedliche, Merkmale.

1) In der Mehrzahl der Fälle tritt die Bronchopneumonie nach den Masern in den ersten 7 Tagen nach dem Beginn des Exanthems auf, und bei einem früheren Einsetzen der Lungenkomplikation ist der Verlauf nicht schwerer.

2) Die klinischen Symptome sind in beiden Gruppen gleich, aber bei denen, die eben Masern gehabt haben, dauern das Fieber und die Lungenerscheinungen laenger an.

3) Bei der Röntgenuntersuchung sind bei den Säuglingen der studiarten Gruppe umschriebene Herde vorherrschend, bei den Kontrollen hingegen mehr diffuse Herde.

4) Das Blutbild bei der Aufnahme und im weiteren Verlauf ist in beiden Fällen ähnlich.

5) Die Bronchopneumonien nach den Masern weisen mehr Komplikationen auf, besonders in den oberen Luftwegen, aber auch auf der Lunge.

6) Die Sterblichkeit ist gering, $4 \%$ in unserer Serie.

7) Die Behandlung der Bronchooneumonie nach den Masern muss in diesem Alter auf Antibioticis mit breitem Wirkungsspektrum, die bessere Erfolgsaussichten bieten, beruhen.

8) Auf Grund all dieser Ueberlegungen glauben wir, dass man an der Diagnose "Branchopneumonie nach Masern" festhalten sollte, nicht im Sinne einer von der bakteriellen Bronchopneumonie des Säuglings verschiedenen Krankheitseinheit, sondern als Hinweis auf die ernstere Prognose. 


\section{BIBLIOGRAFÍA}

1.-MARTINEE, F.; TFAUTMAN, A. Y WIEDENSLADFER, A. - Estudio en 81 crsos de Bronconeumonia Saramplonose. Replsta Chllene de Pediatrí. Ne 5, Mayo de 1955.

2.-STOKES, J. y MTTCHFLI,NTESSON, - Tratada de Pediatrin, Saiva.t. Pástena 871, 1252.

3.-UNDURRAGA, O. Y BAUFI, R. - Bronconeumonía Garamplonosa. Rev. Chllena de Podlatria. No 11-12, Noplembre-Dlctembre de 1954.

4.-BAZAN, C. - Revista Peruane de Pedistria, 1955.

\section{DISCUSTóN}

Prof. Steeger: Se refiere a la mortalidad de las bronconeumonías sarampionosas, suya cifra de $4 \%$ estima de importancia si se considera la alta frecuencia del sarampión.

En el estudio radiológico le ha llamado la atención la localización pseudo-lobar. que se explica porque el sacampión se presenta después de los 6 meses de edad. Ptegunta a los telatores si han encontrado bronconeumonías en el periodo prodrómico, donde alcan. zan caracteres nuy fogaces.
En cuanto al tratamiento. recuerda que en el Hospital Arriarán usaron terramicina en la misma época que el Prof. Ariztia obtenia baenos resultados en sn servicio del Hospital Calvo Mackenna con penicilina y estreptomicina. No se muestra partidario del uso de ia cortisona que puede agtavar estos cuadros. annque reconace que muchos niños al ingreso del Hospital ya vienen tratados con hormonas desde los Centros Periféricos.

Recomienda limitar la época dol lactante hasta el año de edad, cal cono se bace ahora en las estadísticas internacionales.

Dr. Howard: En genetal esta de acuerdo con lo expresado por el Prof. Steeger. También han obseryado bronconeumonías en el período prodrómico. En cuanto a la cortisona, no se usó en estos niños. Consideta que debe limitarse la edad del lactante hasta el año. St prolangación hasta los dos años es an vicio nal. cional derivado de la escuela alemana.

Dr. Montero (Presidente) : Declata incorporado al Dr. Oyanedel a Ja Sociedad Chilena de Pediatria. 